\title{
An integrated continuous downstream process with real-time control: A case study with periodic countercurrent chromatography and continuous virus inactivation
}

\author{
Anton Löfgren ${ }^{1}$, Joaquin Gomis-Fons ${ }^{1}$, Niklas Andersson ${ }^{1}$, Bernt Nilsson ${ }^{2}$, Lotta \\ Berghard $^{3}$, and Chistine Lagerquist Hägglund ${ }^{3}$ \\ ${ }^{1}$ Lunds Universitet Tekniska Hogskola \\ ${ }^{2}$ Lund University \\ ${ }^{3}$ Swedish Orphan Biovitrum AB
}

September 16, 2020

\begin{abstract}
Integrated continuous downstream processes with process analytical technology offer a promising opportunity to reduce production costs and increase process flexibility and adaptability. In this case study, an integrated continuous process was used to purify a recombinant protein on laboratory scale in a two-system setup that can be used as a general downstream setup offering multi-product and multi-purpose manufacturing capabilities. The process consisted of continuous solvent/detergent virus inactivation followed by periodic countercurrent chromatography in the capture step, and a final chromatographic polishing step. A real-time controller was implemented to ensure stable operation by adapting the downstream process to external changes. A concentration disturbance was introduced to test the controller. After the disturbance was applied, the product output recovered within 6 hours, showing the effectiveness of the controller. In a comparison of the process with and without the controller, the product output per cycle increased by $27 \%$, the resin utilization increased from $71.4 \%$ to $87.9 \%$, and the specific buffer consumption was decreased by $21 \%$ with the controller, while maintaining a similar yield and purity as in the process without the disturbance. In addition, the integrated continuous process outperformed the batch process, increasing the productivity by $95 \%$ and the yield by $28 \%$.
\end{abstract}

\section{Introduction}

The increasing need to develop new biopharmaceuticals and reduce costs have led to higher demands on processes being more versatile and efficient (Zydney, 2015). The opportunities and rationale for integrated continuous processes have been discussed in several studies (Hammerschmidt, Tscheliessnig, Sommer, Helk, \& Jungbauer, 2014; Konstantinov \& Cooney, 2015; Papathanasiou \& Kontoravdi, 2020), and integrated continuous processes is also encouraged by the U.S. Food and Drug Administration (FDA) (Woodcock, 2014). There is also a trend towards more stratified therapies, which will lead to an increase in the demand for flexible multi-purpose facilities for smaller-scale production (Gronemeyer, Ditz, \& Strube, 2014).

Upstream processes have historically been the subject of greater development in regard to integration and continuous production than downstream processes (Gronemeyer et al., 2014), despite the fact that up to $60 \%$ of the total manufacturing cost can be attributed to downstream processing (Dileo, Ley, Nixon, \& Chen, 2017). Several methods have been developed to reduce this disparity, including periodic countercurrent chromatography (PCC) (Godawat et al., 2012), multicolumn countercurrent solvent gradient purification 
(Müller-Spath, Aumann, Melter, Strohlein, \& Morbidelli, 2008), and simulated moving bed chromatography (Juza, Mazzotti, \& Morbidelli, 2000). Integrated biomanufacturing has been found to give higher productivity and product quality than non-integrated batch processing (Andersson et al., 2017; Gomis-Fons et al., 2019; Rathore, Agarwal, Sharma, Pathak, \& Muthukumar, 2015; Schugerl \& Hubbuch, 2005). Karst, Steinebach, Soos, and Morbidelli (2017) suggested that the improvement in product quality was the result of a more stable cellular environment and the shorter residence time. Furthermore, the need for manual intervention is reduced, which increases the robustness and reproducibility of the process (Konstantinov \& Cooney, 2015; Sonnleitner, 1997). The FDA advocates the concept of quality by design for the production of biopharmaceuticals (Rathore \& Winkle, 2009). It is therefore important that the process design is retained when the process is scaled up. This is an important reason for developing integrated and continuous processes in research. Another reason for studying integrated and continuous processes is the need for flexibility to allow many similar candidates to be rapidly produced and screened. Many studies on integrated continuous biomanufacturing processes have been performed on monoclonal antibodies (Arnold, Lee, Rucker-Pezzini, \& Lee, 2019; Godawat, Konstantinov, Rohani, \& Warikoo, 2015; Gomis-Fons, Schwarz, et al., 2020; Kamga, Cattaneo, \& Yoon, 2018; Steinebach et al., 2017). This paper describes a general integrated continuous process for the production of various biopharmaceuticals, including a PCC operation and a truly continuous solvent/detergent virus inactivation step (Martins et al., 2019). To the best of our knowledge, this is the first time PCC and continuous virus inactivation have been integrated and run together.

Process analytical technology (PAT) is a tool that enables improved product quality and process efficiency (Fisher et al., 2019), and can be used in the quality-by-design approach. The main goal of PAT is to ensure consistent quality by both a sound understanding of the process and real-time monitoring of critical attributes (Read et al., 2010). Measurements of the attributes can be used for feedback or feedforward process control (Fisher et al., 2019). For example, Mendhe, Thukkaram, Patil, and Rathore (2015) analyzed a variety of PAT-based pooling strategies and found that the most successful application was a feedforward approach based on the retention time of a characteristic peak eluting prior to the main peak. Another example is the implementation of a strategy to control the load factor in a twin-column periodic capture step coupled with a bioreactor for the production of monoclonal antibodies, as in our previous study (Gomis-Fons, Schwarz, et al., 2020), which was achieved by online estimation of the harvest concentration and the model-based assessment of the dynamic binding capacity of the column. This type of control is necessary to avoid overloading the column when the harvest concentration increases, or to maximize the resin utilization when the harvest concentration is low. However, this approach required a very detailed and complex model, which involved substantial experimental work for its calibration. In the present study, we developed a real-time control approach in which no model is needed, and the control parameters are obtained automatically online, thus avoiding expensive and time-consuming experiments. This constitutes a very powerful PAT-based tool that can be used to improve process efficiency and facilitate the efficient integration of upstream and downstream processes, since it allows greater adaptability of the downstream process to changes in the concentration and flow rate from upstream processes.

The supervisory control and data acquisition system Orbit software (Nilsson, Andersson, Gomis-Fons, \& Lofgren, 2017) was used to store the generated data, analyze them, and control the hardware. This system has been used in previous studies (Andersson et al., 2017; Gomis-Fons et al., 2019; Lofgren et al., 2018). Orbit was modified so that the real-time controller could be implemented and to allow online monitoring of process attributes.

\section{The case studied}

The case studied was the purification of a recombinant protein produced by a Chinese hamster ovary cell line, provided by Swedish Orphan Biovitrum AB (Stockholm, Sweden). The purification process was based on the integrated downstream process presented in our previous study (Lofgren et al., 2018). In that study, the

clarified supernatant was captured using a weak anion-exchange column (capture step), followed by a virus 
inactivation step and hydrophobic interaction chromatography (polishing step). In contrast to our previous study, we used a 3-column PCC operation as the capture step with prior continuous virus inactivation, as illustrated in Figure 1, and we implemented a real-time controller to adapt the system to changes in the load concentration.

Two of the three capture columns in the PCC operation are interconnected during the loading phase so that the product breakthrough from the first column is adsorbed on the second column, thus allowing the resin utilization to be increased without reducing the yield (Godawat et al., 2012; Gomis-Fons, Andersson, et al., 2020). At the same time, a third capture column carries out washing, elution, regeneration and equilibration phases (recovery phases). PCC runs periodically in three cycles consisting of two stages each: one in which the columns are interconnected during the loading phase, and the other in which they are interconnected during the washing phase (see Godawat et al., 2012 or Gomis-Fons, Andersson, et al., 2020 for further details). The position of the three capture columns is changed between each cycle.

Four different scenarios were compared to evaluate the performance of the implemented process: the process run in batch mode with the virus inactivation step followed by a single-column capture step and the polishing step (Case "Batch"), the integrated continuous process with constant load concentration (Case c0), and the integrated continuous process with a change in load concentration with and without control (Cases c1A and c1B, respectively). Case c1B was based on an extrapolation of Case c1A after the disturbance.

\section{Material and methods}

\section{Materials}

Three prepacked $1 \mathrm{~mL}$ HiTrap $^{\mathrm{TM}}$ DEAE FF columns were used for the capture step, and a $5 \mathrm{~mL}$ HiTrap $^{\mathrm{TM}}$ Phenyl FF (high sub) column was used in the polishing step (all from Cytiva, Uppsala, Sweden). The buffers in each step were the same as those used in our previous study (Lofgren et al., 2018), and the chromatography columns were sanitized with $1 \mathrm{M} \mathrm{NaOH}$ after each cycle. Conditioning between the two steps was achieved with inline dilution using a high-salt concentration buffer as a conditioning buffer and a $1.4 \mathrm{~mL}$ dynamic mixer. The conductivity and $\mathrm{pH}$ of the conditioning buffer were adjusted to obtain the desired values after inline dilution, thus ensuring product binding on the polishing column. The ratio of eluate volume from the capture column to conditioning buffer volume was 1:1.6.

The continuous virus inactivation reactor was a Cytiva C-type column packed with 150-250 $\mu \mathrm{m}$ silica beads, with an inner diameter of $16 \mathrm{~mm}$ and a maximum height of $400 \mathrm{~mm}$. The clarified supernatant was diluted inline at a ratio of 9:1 with a solvent/detergent mixture containing $10 \mathrm{vol} \%$ Polysorbate 20 and 3 vol\% Tri-n-butyl phosphate, which was loaded from a $50 \mathrm{~mL}$ Superloop $^{\mathrm{TM}}$ (Cytiva). A $1.4 \mathrm{~mL}$ dynamic mixer was used for inline dilution of the supernatant. Cold water was pumped continuously through a cooling jacket to minimize the risk of microbial growth in the reactor, as this could plug the reactor. In addition, the reactor was sanitized with $1 \mathrm{M} \mathrm{NaOH}$ before the start of each run, and was filled with $20 \mathrm{vol} \%$ ethanol for storage.

The integrated continuous downstream process was implemented in two ÄKTA ${ }^{\mathrm{TM}}$ pure 150 systems, controlled by the software UNICORN ${ }^{\mathrm{TM}} 7$, (all from Cytiva, Uppsala, Sweden). One ÄKTA system was used for virus inactivation and the capture step with the PCC operation, and the other ÄKTA system was used for the polishing step. Each ÄKTA system included the following elements: two gradient pumps (pump A and B), a sample pump, several versatile valves (VV) to adjust the flow path, several column and inlet valves, an outlet valve, a fractionator to sample the product, and sensors for measuring UV light absorbance, conductivity, and $\mathrm{pH}$.

An Agilent ${ }^{\mathrm{TM}} 1260$ Infinity II HPLC system (Agilent Technologies Inc., Kista, Sweden) was used for the analytical experiments. The column used for concentration measurements was a Poros ${ }^{\text {TM }} 50 \mathrm{HE}$ column 
(Thermo Fisher Scientific Inc., Stockholm, Sweden) and a Zorbax ${ }^{\text {TM }}$ 300SB-C3 column (Agilent Technologies Inc) for purity.

\section{Process setup}

The setup was an adaptation of that used previously (Gomis-Fons, Andersson, \& Nilsson, 2020); the main difference being the inclusion of continuous virus inactivation with solvent/detergent before the capture step (see Figure 2). In this setup, the capture columns used in the PCC operation were loaded directly from the virus inactivation reactor (red line in Figure 2). The recovery phases were performed with the sample pump (dotted blue line in Figure 2). The eluate from the third capture column was passed through a number of sensors (to measure UV absorption, conductivity, and $\mathrm{pH}$ ) and was then loaded directly onto the polishing column, without a hold-up volume. Two outlet valves allowed the collection of the product in different parts of the downstream process for quantification of the productivity and the yield.

Two pumps were necessary for continuous virus inactivation: one to load the supernatant solution (pump AP), and another to dilute the supernatant inline with the solvent/detergent mixture (pump BP). Pumps $\mathrm{AP}$ and $\mathrm{BP}$ were also used to vary the load concentration by setting different proportions of the flow rate provided by the two pumps. Case $\mathrm{c} 0$ was run with detergent, and Case $\mathrm{c} 1 \mathrm{~A}$ was run with water. The reason why water, instead of detergent, was used in Case c1A was that the load concentration could not be changed in the current setup at the same time as inline dilution with detergent, since pump BP was used for both purposes. Thus, in Case c1A, pump BP was used to dilute the supernatant with water to adjust the load concentration, and in Case c0, pump BP was used to inject the detergent and mix it with the supernatant.

\section{Analytical methods}

A breakthrough curve experiment was performed with an ÄKTA pure 150 system. The supernatant was mixed inline with water at a ratio of 9:1 to emulate the loading conditions in the capture step, and then loaded onto the capture column. The outlet stream was sampled with a fractionator with a sample size of 2 $\mathrm{mL}$. The breakthrough samples, together with a sample of the clarified supernatant, were then analyzed on the HPLC system. For the concentration measurements, aqueous buffers with different salt concentrations were used at a flow rate of $0.5 \mathrm{~mL} / \mathrm{min}$. The absorbance at a detection wavelength of $280 \mathrm{~nm}$ was used to determine the concentration, by using an extinction coefficient of $1.33 \mathrm{~mL}^{-1} \mathrm{mg} \mathrm{cm}^{-1}$, knowing that the path length of the cell was $0.2 \mathrm{~cm}$. Resin utilization, defined as the amount of adsorbed product divided by the maximum amount of adsorbed product at a specific load concentration, was estimated from the breakthrough curve.

A U9-M UV monitor, included in each ÄKTA system, was used to obtain the amount of purified product in each cycle (hereinafter referred to as "product output"). The absorbance at a detection wavelength of $280 \mathrm{~nm}$ was measured and the concentration of the final product, which corresponds to the eluate from the polishing step, was then determined by using the extinction coefficient. The product output was calculated as the concentration multiplied by the eluate volume, which was obtained by multiplying the elution flow rate by the pooling time. The product output was used for the loading control, and to calculate the yield, defined as the product output divided by the amount of product loaded; the productivity, defined as the mass of product output divided by the process time and the total amount of resin in the process; and the specific buffer consumption, defined as the total amount of buffer consumed divided by the product output.

The breakthrough of both the capture and the polishing columns were collected during the run. The eluate from the polishing step was also collected during the whole run, while the eluate from the capture column was only sampled in the fourth cycle, when steady state had been achieved. Reversed-phase chromatography was performed to determine the purity of all these samples. Two buffers with different contents of water and acetonitrile were used (one as equilibration and loading buffer with 5 vol\% acetonitrile, and another as elution buffer with $95 \mathrm{vol} \%$ acetonitrile) at a flow rate of $1 \mathrm{~mL} \mathrm{~min}^{-1}$. The absorbance at a detection 
wavelength of $280 \mathrm{~nm}$ was used to determine the concentration. The purity was then calculated by dividing the area under the peaks corresponding to the pure product by the total area under the peaks.

An $\ddot{A K T A}{ }^{\mathrm{TM}}$ pure 150 system was used to validate the design of the continuous virus inactivation reactor by obtaining a residence time distribution curve. A U9-M UV monitor, included in the same ÄKTA system, was used to detect the product in the outlet of the reactor at a wavelength of $280 \mathrm{~nm}$.

\section{Process design}

\section{PCC design}

The design of the PCC operation was based on the approach described by Godawat et al. (2012), in which several equations corresponding to a continuity constraint and several yield constraints were used to determine the PCC cycle times with the aid of an empirical model of a breakthrough curve. This approach is only valid for a specific flow rate, and different breakthrough curves must thus be obtained at different flow rates. For this reason, the loading flow rate was determined beforehand, and an experimental breakthrough curve was then obtained for this specific flow rate.

The flow rate was calculated by dividing the load volume by the minimum cycle time. The load concentration and volume were $0.74 \mathrm{mg} \mathrm{mL}-1$ and $27 \mathrm{~mL}$, respectively, as in our previous implementation of the process (Löfgren et al., 2018). The cycle time must be equal to, or longer than, the recovery time, based on the continuity constraint (Godawat et al., 2012). In this case, the capture and polishing steps were run simultaneously, and therefore the minimum cycle time was determined by the longest recovery time of these steps, as described previously (Gomis-Fons, Andersson, et al., 2020). By determining the flow rate in this way, at least the same yield and resin utilization as we obtained previously could be obtained at the minimum cycle time (Löfgren et al., 2018). The minimum cycle time was 79 min (the recovery times for the capture and polishing steps were 61 and $79 \mathrm{~min}$, respectively), which led to a flow rate of $0.34 \mathrm{~mL} \mathrm{~min}{ }^{-1}$. As the supernatant was mixed with detergent at a ratio of 9:1, the total incoming flow rate was $0.38 \mathrm{~mL} \mathrm{~min}{ }^{-1}$. If the downstream process were to be connected to a perfusion bioreactor, the load flow rate and concentration would be determined by the harvest stream of the bioreactor, and would therefore be variable. For this reason, the values of flow rate and concentration given above should be considered as nominal design values, and they would correspond to the expected steady-state values from the bioreactor. Therefore, connection to a perfusion bioreactor would be possible by scaling the columns in the downstream process up or down to obtain the desired flow rate and resin volume.

The load volume was then optimized with the breakthrough curve in order to maximize resin utilization and obtain a high yield. Godawat et al. (2012) proposed a method based on the area under the breakthrough curve in the loading phase, in which two columns are interconnected to avoid any loss of product in the breakthrough. The area under the breakthrough curve of the first interconnected column, which corresponds to the amount of product breaking through this column and loaded on the second interconnected column, must be smaller than the dynamic binding capacity, to avoid product breakthrough in the second column. The design in the present study was more flexible, allowing this area to be greater than the dynamic binding capacity, thus some product loss was tolerated in the breakthrough. The concept for the design of the PCC operation based on a breakthrough curve is presented in Figure 3.

When a PCC cycle starts, the first column is already loaded with product from the previous cycle, corresponding to a load volume $V_{1}$. At the end of a PCC cycle, the total loaded volume in a column is $V_{2}$. The load volume in a cycle (hereinafter referred to as $V_{\text {cycle }}$ or "cycle load volume") is therefore equal to $V_{2}-V_{1}$. The area under the curve between $V_{1}$ and $V_{2}\left(k_{1}\right)$ corresponds to the amount of product loaded onto the second column. In other words, the breakthrough of the first column during $V_{\text {cycle }}$ corresponds to a load volume on the second column of $V_{1}$. The area under the curve during $V_{\text {cycle }}\left(k_{1}\right)$ must therefore be equal to the rectangle area between zero and $V_{1}$, which is equivalent to $k_{2}+k_{3}$. The area under the curve between zero and $V_{1}\left(k_{2}\right)$ is the product loss due to breakthrough, and was used to predict the yield by dividing the amount of product adsorbed $\left(k_{3}+k_{4}\right)$ by the total amount loaded in the column $\left(k_{2}+k_{3}+k_{4}\right)$. The resin 
utilization was estimated by dividing the amount of product adsorbed $\left(k_{3}+k_{4}\right)$ in the column by the total binding capacity, estimated $a s k_{3}+k_{4}+k_{5}$.

It can be seen from Figure 3 that increasing the load volume $\left(V_{2}\right)$ increases the resin utilization, but the yield falls as the result of increased product loss at breakthrough in the second column $\left(k_{2}\right)$. There is thus a relationship between resin utilization, yield and load volume, as shown in Figure 4. The resin utilization remains above $70 \%$ for load volumes greater than $35 \mathrm{~mL}$, while the yield is not significantly affected until load volumes of $60-70 \mathrm{~mL}$ are reached. In other words, the resin utilization can be significantly increased by allowing some product loss at breakthrough, as opposed to the approach described by Godawat et al. (2012), in which no product loss is allowed and the yield is therefore set at $100 \%$.

The loss of binding capacity over time, due to column degradation, must be taken into consideration in the design of a chromatography process. An empirical model previously used by Godawat et al. (2012) was used to predict new breakthrough curves with reduced capacities corresponding to 80 and $90 \%$ of the original capacity:

$$
\overline{C=C_{F} \frac{1}{2}\left(1+\operatorname{erf}\left(\frac{x-k_{s}}{\sigma}\right)\right) \quad(1)}
$$

where $C$ and $C_{F}$ are the outlet and feed concentrations in $\mathrm{mg} \mathrm{mL}^{-1}$, respectively, erf is the error function, $x$ is the loaded mass in $\mathrm{mg}$ per $\mathrm{mL}$ resin, $k_{s}$ is the binding capacity in $\mathrm{mg}$ per $\mathrm{mL}$ resin, and $\sigma$ is an empirical dimensionless constant.

New yields were obtained with this model with the same amount of adsorbed product as in the original scenario with $100 \%$ capacity. In other words, we studied how the yield was affected by reduced binding capacity at constant amount of adsorbed product in the capture step. Loss of binding capacity does not affect the yield for low load volumes (see Figure 4), but the yield decreases at load volumes of 35 and 45 $\mathrm{mL}$ for the $80 \%$ and $90 \%$ capacity scenarios, respectively. It is not possible to obtain the same amount of adsorbed product in these scenarios with capacity loss by loading more product, which is why yields are not shown in these cases at higher load volumes. For example, at a load volume of $40 \mathrm{~mL}$, where the resin utilization is around $83 \%$, it would not be possible to maintain the amount of adsorbed product if the capacity decreased $20 \%$, as the maximum attainable amount of adsorbed product would be $80 \%$ of the original resin capacity, but the desired amount would be $83 \%$ of the original resin capacity.

The design criterion was to maintain product output with the loading controller while taking a loss of resin capacity into account. Thus, the feasible load volumes are those that are lower than 35 or $45 \mathrm{~mL}$, depending on whether a capacity loss of $20 \%$ or $10 \%$ is assumed, to ensure constant amount of adsorbed product in the capture step and therefore constant product output. A $10 \%$ loss in resin capacity was assumed in this study in the design of the PCC operation. The load volume was therefore set at $45 \mathrm{~mL}$, which corresponds to a cycle load volume of $36 \mathrm{~mL}$. The cycle time is then $95 \mathrm{~min}$ with a load flow rate of $0.38 \mathrm{~mL} \mathrm{~min}{ }^{-1}$. This fulfills the constraint of the minimum cycle time, which was 79 min.

\section{Continuous virus inactivation}

In the downstream process we used previously (Löfgren et al., 2018), virus inactivation was performed at low $\mathrm{pH}$ after the capture step. In the present study, it was performed before the capture step to allow continuous flow through the virus inactivation reactor, as the inlet stream to the capture step is continuous, but not the outlet stream. Since the input to the capture column must have neutral $\mathrm{pH}$, virus inactivation at low $\mathrm{pH}$ would involve two subsequent dilution steps to decrease and then increase the $\mathrm{pH}$. This would require an extra pump and an extra mixer, and the total volume to be treated by the capture column would be higher. For this reason, solvent/detergent virus inactivation, based on the process presented by Martins et al. (2019), was used instead. Performing virus inactivation before the capture step allowed the detergent to be removed from the product in two consecutive steps. 
The continuous virus inactivation reactor was designed to ensure a minimum product residence time of 60 min. The mean residence time is given by dividing the void volume by the flow rate. The design void volume was calculated by multiplying the residence time by the loading flow rate $\left(0.38 \mathrm{~mL} \mathrm{~min}{ }^{-1}\right)$ and adding an extra $20 \%$ to account for dispersion. The void fraction was assumed to be 0.36 , as estimated by de Klerk (2003) for a packed bed with a high column to particle diameter ratio. Then, the total reactor volume was $76.0 \mathrm{~mL}$, and the height was $378 \mathrm{~mm}$. An experiment was run with supernatant to check the residence time distribution and validate the design. It was found that $99.8 \%$ of the product remained in the reactor for more than $60 \mathrm{~min}$, which is higher than the $99 \%$ suggested by Martins et al. (2019). The mean residence time was $75.5 \mathrm{~min}$, and the ratio between the residence times at $50 \%$ and $1 \%$ of the cumulative residence time distribution curves was 1.16; 1 being the ideal value, i.e. equal residence time for all the molecules. This value is similar to that obtained by Martins et al. (2019) in a small-scale packed-bed reactor. A portion of the product $(4 \%)$ resided in the reactor for more than $100 \mathrm{~min}$. In low-pH virus inactivation, such a long residence time could have led to the risk of the formation of aggregates; however, such a risk has not been reported in a solvent/detergent virus inactivation process.

\section{Column design}

The column design was based on the approach described by us previously (Gomis-Fons et al., 2019), where it was shown that several constraints must be fulfilled for the integration of the columns. One of these is related to the column capacities, i.e., the polishing column must have sufficient capacity to handle the product eluted from the capture column. As it was desirable to keep the scale of the process as small as possible, the capture column volume was set to the minimum possible, $1 \mathrm{~mL}$, and the polishing column volume was calculated based on its capacity and the amount of product per cycle. The concentration of the supernatant diluted with the detergent was $0.74 \mathrm{mg} \mathrm{mL}^{-1}$, and the volume loaded in a column was $45 \mathrm{~mL}$, as mentioned above. Therefore, the maximum amount of product loaded on the polishing column per cycle was $33.3 \mathrm{mg}$. The capacity of that column was approximately $10 \mathrm{mg}$ per $\mathrm{mL}$ column, thus leading to a minimum column volume of $3.3 \mathrm{~mL}$. Due to the limited availability of volumes in prepacked columns, a $5 \mathrm{~mL}$ column was chosen.

Another constraint that must fulfilled for the successful integration of the columns is related to the residence times, i.e., the residence time in the columns must be equal to, or longer than, a certain reference value in each phase, which, in this case, was obtained from our previous study (Löfgren et al., 2018). Therefore, the residence time was set equal to the reference value in all the phases, except for the loading phase of the polishing step. In this phase, the flow rate, which is directly related to the residence time, is given by the flow rate in the elution phase of the capture step $\left(0.55 \mathrm{~mL} \mathrm{~min}^{-1}\right)$ plus the flow rate of the conditioning stream $\left(0.88 \mathrm{~mL} \mathrm{~min}{ }^{-1}\right)$. The load flow rate was thus $1.43 \mathrm{~mL} \mathrm{~min}-1$, and the residence time was $3.5 \mathrm{~min}$, which was longer than the reference value for this phase, of $2 \mathrm{~min}$.

\section{Process control}

\section{Control software}

The ÄKTA pure systems were controlled by external software called Orbit (Nilsson et al., 2017). The software is written in the general-purpose, object-oriented scripting language Python 3.7. Orbit sends instructions to UNICORN sequentially, and UNICORN forwards them to the ÄKTA system. Separate Orbit programs were used for each system. Synchronization between and within the systems was achieved through event flags. For example, the system in which the polishing step is performed sends a flag when this column is ready to be loaded, and the other system sends another flag when the elution phase is about to start in the capture step; the elution phase starts when both systems have sent their respective flags. (Further details on the control of multi-system setups by Orbit can be found in Gomis-Fons, Andersson, et al., 2020). The control strategies were implemented in the Orbit programs, for which process data, such as the cycle load volume or the UV absorbance, was monitored online. 


\section{Loading control}

As the feed concentration and flow rate from a bioreactor can vary over time, a real-time iterative learning controller was implemented to adapt the system to these changes (Figure 5). The aim was that the same amount of product would be purified in the downstream process for every PCC cycle, i.e. to maintain a constant product output. The cycle load volume was modified to control the amount of product purified in a cycle. For example, if the concentration was lower than the nominal value used in the design, the product output would be lower. In order to correct for this, the volume loaded onto the capture step would be increased by extending the PCC cycle, so that the product output became higher in the next cycle. The control software Orbit tracked the loading flow rate, which allowed obtaining the right PCC cycle time to achieve the desired cycle load volume.

Iterative learning control is an approach to minimize the difference between the desired value and the measured value in systems that operate in a repetitive mode (Longman, 2000). Control was based on the so-called previous cycle learning scheme (Ouyang \& Pipatpaibul, 2010; Xu, Heng Lee, \& Zhang, 2004), since only the signals from the previous iteration are used to obtain the control signal for the next iteration (see Figure 5). A learning control law was used to update the control signal, as shown in Eq. (2). In this learning control law, the control signal at iteration $k\left(u_{k}\right)$ depends on the control signal at the previous iteration $\left(u_{k-1}\right)$, the tracking error between the reference $\left(y_{\text {ref }}\right)$ and the output signal at the previous iteration $\left(y_{k-1}\right)$, and the controller gain $(K)$.

$$
\overline{u_{k}=u_{k-1}+K\left(y_{\mathrm{ref}}-y_{k-1}\right) \quad(2)}
$$

In this case, the control signal is the cycle load volume in units of $\mathrm{mL}$, and the output signal is the product output in units of mg. The relationship between these signals can be approximated by a linear expression that depends on a transfer function of the process $(G)$, and neglects system uncertainties and measurement errors.

$$
\overline{y_{k}=\mathrm{Gu}_{k} \quad(3)}
$$

The design criterion for the selection of the controller gain is the convergence, i.e., the tracking error should tend to zero, and the condition for this is (Xu et al., 2004):

$$
\overline{\|1-G K\|=a<1 \quad(4)}
$$

Therefore, the controller gain can be defined as a function of a parameter $(a)$, which has values between zero and one.

$$
\overline{K=(1-a) G^{-1} \quad(5)}
$$

When the value of $a$ is close to one, the controller response will be slower because the controller gain decreases, but the controller will be more robust to system uncertainties and measurement errors, since it is far from being in the instable region defined by Eq. (4). If $a$ is close to zero, the controller will respond more quickly, but there will be a higher risk of oscillations and divergence. In the extreme case, when $a$ equals zero, called the deadbeat scenario, convergence is achieved after one iteration, but an exact model of the system is required to predict the transfer function, and there must be no external disturbances or measurement errors (Xu \& Tan, 2002). In the present study, the parameter $a$ was set to 0.3 .

The transfer function was updated online for each cycle during the experiment, i.e., the transfer function 
was calculated after each iteration with the values obtained for $u_{k-1}$ and $y_{k-1}$, using Eq. (3). The controller gain was also updated for each cycle as this depends on the transfer function. This allows correction for possible disturbances in the downstream processing, such as loss of capacity of the columns or changes in the concentration or flow rate of the feed. Therefore, the speed of the controller was adjusted depending on the variation in the transfer function. Combining Eq. (5) with Eq. (3) allows the learning control law in Eq. (2) to be re-written as follows:

$$
\overline{u_{k}=u_{k-1}\left(a+(1-a) \frac{y_{\mathrm{ref}}}{y_{k-1}}\right) \quad(6)}
$$

The reference output $\left(y_{\text {ref }}\right)$ was also determined online during the experiment after process startup. The previously calculated PCC cycle time was applied during the first few cycles until steady state had been reached. The area of the eluate peak from the polishing step was then automatically calculated and was used to calculate the product output, which was set as the reference signal.

\section{Pooling control}

The cutoff times of the pooling of the product in the polishing step were obtained based on the UV absorbance at a wavelength of $280 \mathrm{~nm}$, measured online with a U9-M UV monitor, i.e., pooling started and ended at a certain value of absorbance, as described previously (Löfgren et al., 2018). In addition, in the present study, the second absorbance cutoff (i.e., when pooling ends) was automatically adjusted to compensate for the variation in the incoming concentration. The height of the eluate peak was obtained online, and the absorbance cutoff was calculated based on the height. Previous runs of the process were used to determine the linear relationship between the absorbance cutoff and the peak height.

\section{Results and discussion}

\section{Experimental validation}

The complete integrated continuous downstream process was run on laboratory scale with the process setup presented in Figure 2. If the downstream process were connected to a perfusion bioreactor, the load concentration would vary over time. Therefore, in order to test the performance of the control strategies, an external disturbance was introduced in the form of a $20 \%$ decrease in the load concentration, to emulate a change in the harvest concentration that would occur during a perfusion run.

The chromatogram shown in Figure 6 corresponds to a run with 9 cycles, including an initial loading stage for process startup. After startup, steady product output was achieved by cycle 4, after running the process with a cycle load volume of $36 \mathrm{~mL}$. The reference value obtained for the product output used in the loading control was $23.1 \mathrm{mg}$. The load concentration was reduced by $20 \%$ at the beginning of cycle 4 , and the effects in the final product were seen after a further two cycles due to the residence time in the virus inactivation loop, as well as the delay between the capture and polishing steps. The measured product output decreased to $18.0 \mathrm{mg}$, and as this value differed from the reference value, the loading controller provided a new cycle load volume proportional to the decrease in concentration. This led to a gradual increase in the cycle load volume from $36 \mathrm{~mL}$ in cycle 5 , to $44.6 \mathrm{~mL}$ in cycle 9 , which resulted in an increase in product output until a value similar to that of the reference value was reached. In fact, the product output had already reached $22.3 \mathrm{mg}$ only one cycle after the disturbance was detected in the pool of the final product. This means that the product output had almost reached its original value approximately 2 hours after the disturbance was detected in the final product, thus confirming the effective implementation of the loading controller with a rapid response. In a typical perfusion run, a change in concentration of $20 \%$ would be made over a much longer time, thus giving sufficient time for the controller to adjust the cycle load volume to maintain the desired product output. The pooling strategy was also successfully validated, since the absorbance cutoff for 
the end of product pooling in the polishing step was adjusted according to the peak height, as shown in Figure 7 .

The effect of the controller on the response of the downstream process is shown in Figure 8. At the new steady state following the disturbance, implementing the controller led to an increase in the product output of $21.8 \%$ per cycle (Case c1A) compared to the process without the controller (Case c1B), while the cycle load volume increased by $23.9 \%$. The process was run without any disturbance (Case $\mathrm{c} 0$ ) to provide a baseline to compare the process response with and without the disturbance.

An external disturbance in the load flow rate was considered, but not investigated experimentally. The reason for this was that a change in flow rate would not affect the process as the load volume was continuously tracked by the control software Orbit. In other words, if the flow rate was reduced, the cycle time would be extended, but the cycle load volume would be unaffected, thus having no impact on the product output.

\section{Process performance indicators}

Table 1 presents the values of several process performance indicators at steady state for the 4 scenarios studied. The batch process, which was run at a load volume of $45 \mathrm{~mL}$ to achieve the same resin utilization as in the continuous process, had a yield of $68.3 \%$, which is significantly lower than the $87.5 \%$ obtained in the continuous process (Case c0). This is because there is no interconnection between the columns during the loading phase of the capture step, which leads to a higher product loss due to breakthrough. The product output was similar in both cases (22.6 mg in Case "Batch", compared to $23.5 \mathrm{mg}$ in Case c0) since the total amount of product loaded onto the capture column was the same. Therefore, the specific buffer consumption is also close to the value obtained in the continuous process $\left(16.7 \mathrm{~mL} \mathrm{mg}^{-1}\right.$ compared to $16.0 \mathrm{~mL} \mathrm{mg}^{-1} \mathrm{in}_{\text {Case }}$ $\mathrm{c} 0$ ). Three parameters affect the productivity: the product output, the process time and the total volume of resin. The higher the product output and the lower the process time and the resin volume, the higher the productivity. On the one hand, the process time in the batch process was higher (240 min compared to 95 $\min$ ) due to the fact that the loading phase and the recovery phases were not carried out simultaneously, unlike in the PCC operation. On the other hand, the resin volume in the batch process was $6 \mathrm{~mL}(1 \mathrm{~mL}$ of capture resin $+5 \mathrm{~mL}$ of polishing resin), which was less than in the continuous process $(8 \mathrm{~mL})$ because, in this process, 3 columns were used in the capture step due to the PCC operation. In this case, the difference in the process time had a much greater effect than the difference in the resin volume, which led to a significantly lower productivity in the batch process $\left(22.6 \mathrm{mg}^{\text {day }}{ }^{-1} \mathrm{~mL}^{-1}\right)$ in comparison to the continuous process (44.1 $\mathrm{mg}$ day $\left.^{-1} \mathrm{~mL}^{-1}\right)$, as can be seen in Table 1 .

In an evaluation of the effect of the controller on the performance indicators, it was found that the amount of purified product per cycle was higher in the process with control (Case c1A) than in the process without control (Case c1B). This, in turn, led to an increase in resin utilization of $24 \%$ (from $71.4 \%$ to $87.9 \%$ ), as estimated from the breakthrough curve, as more product was purified in each cycle compared to the process without the controller. In addition, the resin utilization and the product output in Case c1A had similar values to those of the process with no external disturbance. Another effect of the higher product output per cycle was the decrease in the specific buffer consumption, i.e., the volume of buffer consumed to purify a certain mass of product. The reduction in specific buffer consumption was estimated to be $21 \%$ compared to the process without the controller. The productivity was lower in Cases c1A and c1B than in Case c0, as the concentration decreased, leading to a lower amount of product being purified per unit time. Regarding the yield, $87.5 \%$ of the incoming protein was recovered in the final product in the case without disturbance. When the load concentration was decreased, the yield was not negatively affected, as the yield was $87.2 \%$ in Case $\mathrm{c} 1 \mathrm{~A}$ and $85.1 \%$ in Case c1B. If the disturbance had been a concentration increase instead, the control system would have reduced the PCC cycle length until the minimum cycle time was reached (79 min), and the yield would have been similar. In contrast, in the process without control, the cycle length would have remained the same, and the yield in the loading of the capture step would have decreased significantly.

The purity was analyzed at different stages of the process in Case c0 to ensure product quality, and the 
results are given in the Supplementary Material, where it can be seen the purity of the product increased after each chromatography step. The purity of the final product was $99.1 \%$. The main impurities were present in the breakthrough of the capture step, as shown in the chromatograms corresponding to the supernatant (Figure S1 in Supplementary Material), the capture pool (Figure S4), and the capture breakthrough (Figure S3). A significant amount of impurities was also present in the breakthrough of the polishing step (Figure S5). The chromatogram of the pure product (Figure S6) did not contain any of the peaks corresponding to the detergent (Figure S2), thus showing that the detergent was effectively removed in the process. Product purity was not measured in Case c1A, but the only difference between Case $\mathrm{c} 0$ and $\mathrm{c} 1 \mathrm{~A}$ was the change in the load concentration, while the impurity profile was the same. It is therefore reasonable to assume that the purity would have remained unchanged in Case c1A.

\section{Conclusions}

An integrated continuous downstream process with PCC and continuous virus inactivation with detergent was successfully implemented in a two-system setup in a case study of the purification of a recombinant protein on laboratory scale. The 3-column PCC operation was designed with minimal experimental effort as only a breakthrough curve was necessary to obtain the required flow rates and cycles times. The residence time distribution in the continuous virus inactivation step was obtained experimentally to check that the protein had a minimum residence time of $60 \mathrm{~min}$. A simple yet effective real-time loading controller based on iterative learning control was implemented to allow adaptation of the downstream process to a change in the load concentration. After a $20 \%$ decrease in concentration, the cycle length was automatically adjusted, giving almost the same product output as before the disturbance in only 2 hours, thus showing the effectiveness of the controller in ensuring a consistent output with rapid response. In addition, a real-time pooling strategy allowed adaptation of the pooling absorbance cutoff levels to the size of the peak in the elution of the polishing step.

It was shown that the continuous process outperformed the batch process, with a productivity increase of $95 \%$ and a $28 \%$ higher yield. Additionally, by comparing the integrated continuous process with and without the controller after the concentration disturbance, it was found that the amount of product loaded was lower in the process without the controller, thus leading to a lower resin utilization and lower product output. This, in turn, led to a higher specific buffer consumption than in the process with the controller. The controller thus ensured similar resin utilization and product output with and without the disturbance.

The whole downstream process was implemented in a two-system setup with minimal use of resources and space. This process setup and the control strategies developed could be used for any downstream process. This configuration is thus well suited in a ballroom-like facility that offers multi-product and multi-purpose manufacturing capabilities, allowing quick responses to market demands.

\section{Declaration of Competing Interest}

The authors declare that they have no conflicts of interest.

\section{Acknowledgements}

The authors acknowledge that this research is part of the Competence Centre for Advanced BioProduction by Continuous Processing (AdBIOPRO), which is co-funded by VINNOVA, the Swedish Agency for Innovation (Grant no. 2016-05181) and the industrials partners: Swedish Orphan Biovitrum, Cobra Biologics, BioInvent, Cytiva, Valneva, Lab-on-a-Bead, and CellProtect Nordic Pharmaceutical. 


\section{References}

Andersson, N., Lofgren, A., Olofsson, M., Sellberg, A., Nilsson, B., \& Tiainen, P. (2017). Design and control of integrated chromatography column sequences. Biotechnology Progress, 33 , 923-930. doi:10.1002/btpr.2434

Arnold, L., Lee, K., Rucker-Pezzini, J., \& Lee, J. H. (2019). Implementation of Fully Integrated Continuous Antibody Processing: Effects on Productivity and COGm. Biotechnology Journal, 14, 1800061. doi:10.1002/biot.201800061

de Klerk, A. (2003). Voidage variation in packed beds at small column to particle diameter ratio. AIChE Journal, 49 , 2022-2029. doi:10.1002/aic.690490812

Dileo, M., Ley, A., Nixon, A. E., \& Chen, J. (2017). Choices of capture chromatography technology in antibody manufacturing processes.Journal of Chromatography B, 1068-1069 , 136-148. doi:10.1016/j.jchromb.2017.09.050

Fisher, A. C., Kamga, M. H., Agarabi, C., Brorson, K., Lee, S. L., \& Yoon, S. (2019). The Current Scientific and Regulatory Landscape in Advancing Integrated Continuous Biopharmaceutical Manufacturing. Trends in Biotechnology, 37 , 253-267. doi:10.1016/j.tibtech.2018.08.008

Godawat, R., Brower, K., Jain, S., Konstantinov, K., Riske, F., \& Warikoo, V. (2012). Periodic countercurrent chromatography - design and operational considerations for integrated and continuous purification of proteins. Biotechnology Journal, 7 , 1496-1508. doi:10.1002/biot.201200068

Godawat, R., Konstantinov, K., Rohani, M., \& Warikoo, V. (2015). End-to-end integrated fully continuous production of recombinant monoclonal antibodies. Journal of Biotechnology, 213 (Supplement C), 13-19. doi:10.1016/j.jbiotec.2015.06.393

Gomis-Fons, J., Andersson, N., \& Nilsson, B. (2020). Optimization study on periodic counter-current chromatography integrated in a monoclonal antibody downstream process. Journal of Chromatography A, 1621 , 461055. doi:10.1016/j.chroma.2020.461055

Gomis-Fons, J., Lofgren, A., Andersson, N., Nilsson, B., Berghard, L., \& Wood, S. (2019). Integration of a complete downstream process for the automated lab-scale production of a recombinant protein. Journal of Biotechnology, 301 , 45-51. doi:10.1016/j.jbiotec.2019.05.013

Gomis-Fons, J., Schwarz, H., Zhang, L., Andersson, N., Nilsson, B., Castan, A., . . . Chotteau, V. (2020). Model-based design and control of a small-scale integrated continuous end-to-end mAb platform.Biotechnology Progress, 36, e2995. doi:10.1002/btpr.2995

Gronemeyer, P., Ditz, R., \& Strube, J. (2014). Trends in Upstream and Downstream Process Development for Antibody Manufacturing.Bioengineering (Basel), 1 , 188-212. doi:10.3390/bioengineering1040188

Hammerschmidt, N., Tscheliessnig, A., Sommer, R., Helk, B., \& Jungbauer, A. (2014). Economics of recombinant antibody production processes at various scales: Industry-standard compared to continuous precipitation. Biotechnology Journal, 9 , 766-775. doi:10.1002/biot.201300480

Juza, M., Mazzotti, M., \& Morbidelli, M. (2000). Simulated moving-bed chromatography and its application to chirotechnology. Trends in Biotechnology, 18 , 108-118. doi:10.1016/S0167-7799(99)01419-5

Kamga, M.-H., Cattaneo, M., \& Yoon, S. (2018). Integrated continuous biomanufacturing platform with ATF perfusion and one column chromatography operation for optimum resin utilization and productivity.Preparative Biochemistry and Biotechnology, 48 , 383-390. doi:10.1080/10826068.2018.1446151

Karst, D. J., Steinebach, F., Soos, M., \& Morbidelli, M. (2017). Process performance and product quality in an integrated continuous antibody production process. Biotechnology and Bioengineering, 114 , 298-307. doi:10.1002/bit.26069 
Konstantinov, K. B., \& Cooney, C. L. (2015). White Paper on Continuous Bioprocessing May 20212014 Continuous Manufacturing Symposium.Journal of Pharmaceutical Sciences, 104 , 813-820. doi:10.1002/jps.24268

Lofgren, A., Andersson, N., Sellberg, A., Nilsson, B., Lofgren, M., \& Wood, S. (2018). Designing an Autonomous Integrated Downstream Sequence From a Batch Separation Process - An Industrial Case Study.Biotechnology Journal, 13, 1700691.doi:10.1002/biot.201700691

Longman, R. W. (2000). Iterative learning control and repetitive control for engineering practice. International Journal of Control, 73 , 930-954. doi:10.1080/002071700405905

Martins, D. L., Sencar, J., Hammerschmidt, N., Tille, B., Kinderman, J., Kreil, T. R., \& Jungbauer, A. (2019). Continuous Solvent/Detergent Virus Inactivation Using a Packed-Bed Reactor. Biotechnology Journal, 14 , 1800646. doi:10.1002/biot.201800646

Mendhe, R., Thukkaram, M., Patil, N., \& Rathore, A. S. (2015). Comparison of PAT based approaches for making real-time pooling decisions for process chromatography-use of feed forward control.Journal of Chemical Technology and Biotechnology, 90 , 341-348. doi:10.1002/jctb.4448

Muller-Spath, T., Aumann, L., Melter, L., Strohlein, G., \& Morbidelli, M. (2008). Chromatographic separation of three monoclonal antibody variants using multicolumn countercurrent solvent gradient purification (MCSGP). Biotechnology and Bioengineering, 100 , 1166-1177. doi:10.1002/jctb.4448

Nilsson, B., Andersson, N., Gomis-Fons, J., \& Lofgren, A. (2017).Supervisory control of integrated continuous downstream processes. Poster presented at the Integrated Continuous Biomanufacturing III, Cascais, Portugal. Retrieved from https://dc.engconfintl.org/biomanufact_iii/80

Ouyang, P. R., \& Pipatpaibul, P. (2010). Iterative Learning Control: A Comparison Study. Paper presented at the ASME 2010 International Mechanical Engineering Congress and Exposition, Vancouver, British Columbia, Canada. doi:10.1115/IMECE2010-37161

Papathanasiou, M. M., \& Kontoravdi, C. (2020). Engineering challenges in therapeutic protein product and process design. Current Opinion in Chemical Engineering, 27 , 81-88. doi:10.1016/j.coche.2019.11.010

Rathore, A. S., Agarwal, H., Sharma, A. K., Pathak, M. \& Muthukumar, S. (2015). Continuous processing for production of biopharmaceuticals.Preparative Biochemistry and Biotechnology, 45, 836-849. doi:10.1080/10826068.2014.985834

Rathore, A. S., \& Winkle, H. (2009). Quality by design for biopharmaceuticals. Nature Biotechnology, 27 , 26-34. doi:10.1038/nbt0109-26

Read, E., Park, J., Shah, R., Riley, B., Brorson, K., \& Rathore, A. (2010). Process analytical technology (PAT) for biopharmaceutical products: Part I. Concepts and applications. Biotechnology and Bioengineering, 105, 276-284. doi:10.1002/bit.22528

Schugerl, K., \& Hubbuch, J. (2005). Integrated bioprocesses.Current Opinion in Microbiology, 8 , 294-300. doi:10.1016/j.mib.2005.01.002

Sonnleitner, B. (1997). Bioprocess automation and bioprocess design. Journal of Biotechnology, 52 , 175-179. doi:10.1016/S0168-1656(96)01642-2

Steinebach, F., Ulmer, N., Wolf, M., Decker, L., Schneider, V., Walchli, R., . . . Morbidelli, M. (2017). Design and operation of a continuous integrated monoclonal antibody production process. Biotechnology Progress, 33 , 1303-1313. doi:10.1002/btpr.2522

Woodcock, J. (2014). Modernizing pharmaceutical manufacturing-continuous manufacturing as a key enabler. Lecture presented at the MIT-CMAC International Symposium on Continuous Manufacturing of Pharmaceuticals, Cambridge, MA, USA. Retrieved from https://iscmp2014.mit.edu/sites/default/files/documents/ISCMP2014_Keynote_Slides.pdf 
Xu, J.-X., Heng Lee, T., \& Zhang, H.-W. (2004). Analysis and comparison of iterative learning control schemes. Engineering Applications of Artificial Intelligence, 17 , 675-686. doi:10.1016/j.engappai.2004.08.002

Xu, J.-X., \& Tan, Y. (2002). Robust optimal design and convergence properties analysis of iterative learning control approaches.Automatica, 38 , 1867-1880. doi:10.1016/S0005-1098(02)00143-7

Zydney, A. L. (2015). Perspectives on integrated continuous bioprocessing - opportunities and challenges. Current Opinion in Chemical Engineering, 10 (Supplement C), 8-13. doi:10.1016/j.coche.2015.07.005

\section{Tables}

Table 1 . Process performance indicators in different process scenarios

\begin{tabular}{lllll}
\hline Parameter & Units & Process scenarios & Process scenarios $^{+}$ & Process scenarios $^{+}$ \\
\hline & & Batch & Case c0 & Case c1A \\
Product output per cycle & $\mathrm{mg}$ & 22.6 & 23.5 & 22.9 \\
Resin utilization & $\mathrm{wt} \%$ & 89.2 & 89.2 & 87.9 \\
Productivity & $\mathrm{mg} \mathrm{day}{ }^{-1} \mathrm{~mL}^{-1}$ & 22.6 & 44.1 & 35.1 \\
Yield & $\mathrm{wt} \%$ & 68.3 & 87.5 & 87.2 \\
Specific buffer consumption & $\mathrm{mL} \mathrm{mg}$ & 16.0 & 16.5 \\
\hline
\end{tabular}

${ }^{+}$Batch: Batch process with virus inactivation followed by a single-column capture step and the polishing step; Case c0: Continuous process with controller and without disturbance; Case c1A: Continuous process with controller and with disturbance; Case c1B: Continuous process without controller and with disturbance.

\section{Figure captions}

Figure 1. Block diagram of the integrated continuous process studied. The clarified supernatant is mixed with detergent and loaded onto the virus inactivation (VI) reactor. The output stream from the reactor is loaded onto two of the capture columns ( $\mathrm{C} 1$ and $\mathrm{C} 2)$ run with a PCC operation. The periodic eluate from one of the capture columns (C3) is conditioned inline and directly loaded onto the polishing column (P)

Figure 2. The two-system setup. The system on the left was used for the continuous virus inactivation and the capture step run with a PCC operation. The system on the right illustrates the polishing step. The red line indicates the flow path during the interconnected load phase of capture column 1 (C1) with the breakthrough going to capture column $2(\mathrm{C} 2)$. The blue dotted line shows the flow path during elution of the fully loaded column 3 (C3) and the simultaneous loading of the polishing column (P). The gray lines show inactive flow paths. The versatile valves (VV) were used to select different flow paths, the loop valve $(\mathrm{LV})$ directs the flow to one of the three capture columns, the column valve (CV) allows the diluted feed to pass through the virus inactivation reactor (VI), and two outlet valves (OutV) allowed product collection in different parts of the process. In the system on the left, Pumps AP and BP were used to load the supernatant onto the capture columns, and the sample pump (SampP) was used in the recovery phases of the capture columns. In the system on the right, the gradient pump (GradP) was used in the recovery phases of the polishing column, and the sample pump was used for the inline conditioning between the two steps.

Figure 3. Areas under the breakthrough curve in relation to the loaded volume.

Figure 4. Effect of the load volume on the resin utilization and yield for different scenarios with respect to the binding capacity in the capture column. Data in the graph correspond to an empirical model (Eq. 
(1)) calibrated with the experimental breakthrough curve. Load volume refers to the total volume loaded onto a column in the $100 \%$ capacity scenario. Yields for $80 \%$ and $90 \%$ capacity scenarios were calculated for loadings with the same amount of adsorbed product as that in the $100 \%$ capacity scenario.

Figure 5. Illustration of the iterative learning control strategy with a previous cycle learning scheme. The variables $u_{k}$ and $y_{k}$ are the control and output signals, respectively, at iteration $k$; and $y_{\text {ref }}$ is the reference value of the output signal.

Figure 6. Upper panel: Chromatographic profile of the capture step including the UV absorbance signal from the breakthrough stream from the interconnected capture columns (UV Breakthrough) and the outlet stream from a capture column during the recovery phases (UV Recovery). Alternate PCC cycles are indicated by light and dark gray backgrounds. Lower panel: Chromatographic profile of the polishing step where the shaded peaks represent the product pool.

Figure 7 . Product pools in cycles 3 and 6 showing the absorbance cutoffs, obtained with the pooling control strategy based on the peak height.

Figure 8 . Effect of the controller on the evolution of the downstream process. The process in which the controller was implemented following a concentration disturbance $(\mathrm{c} 1 \mathrm{~A})$ is compared to a process without disturbance but with the controller $(\mathrm{c} 0)$, and a process with disturbance but without the controller $(\mathrm{c} 1 \mathrm{~B})$.

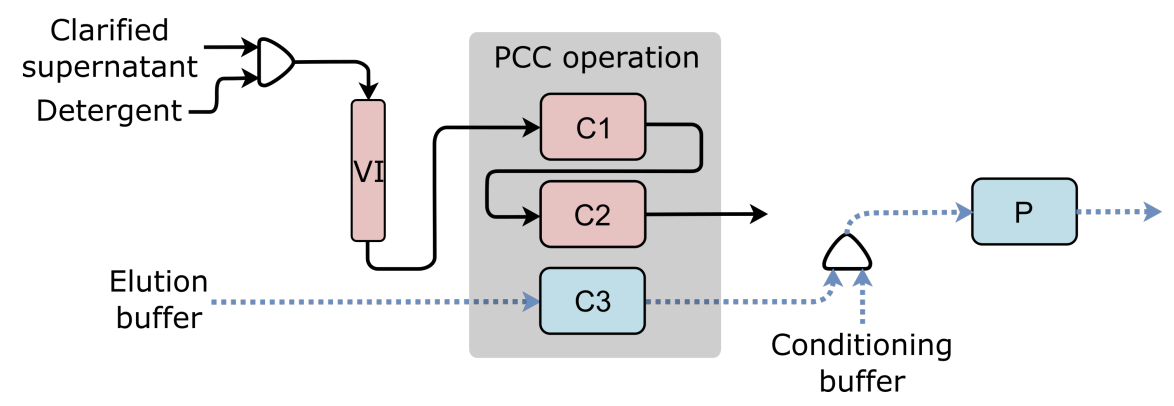



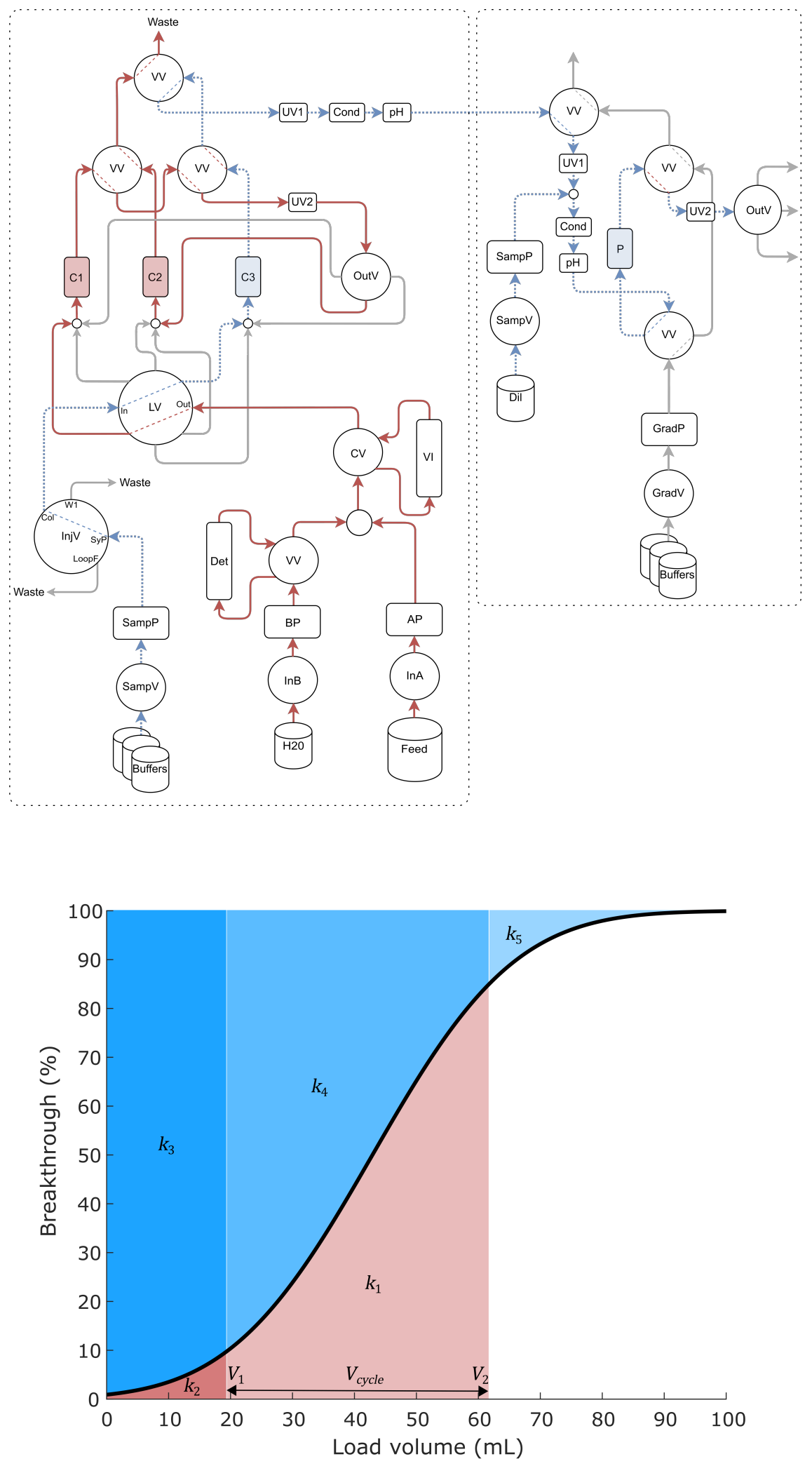

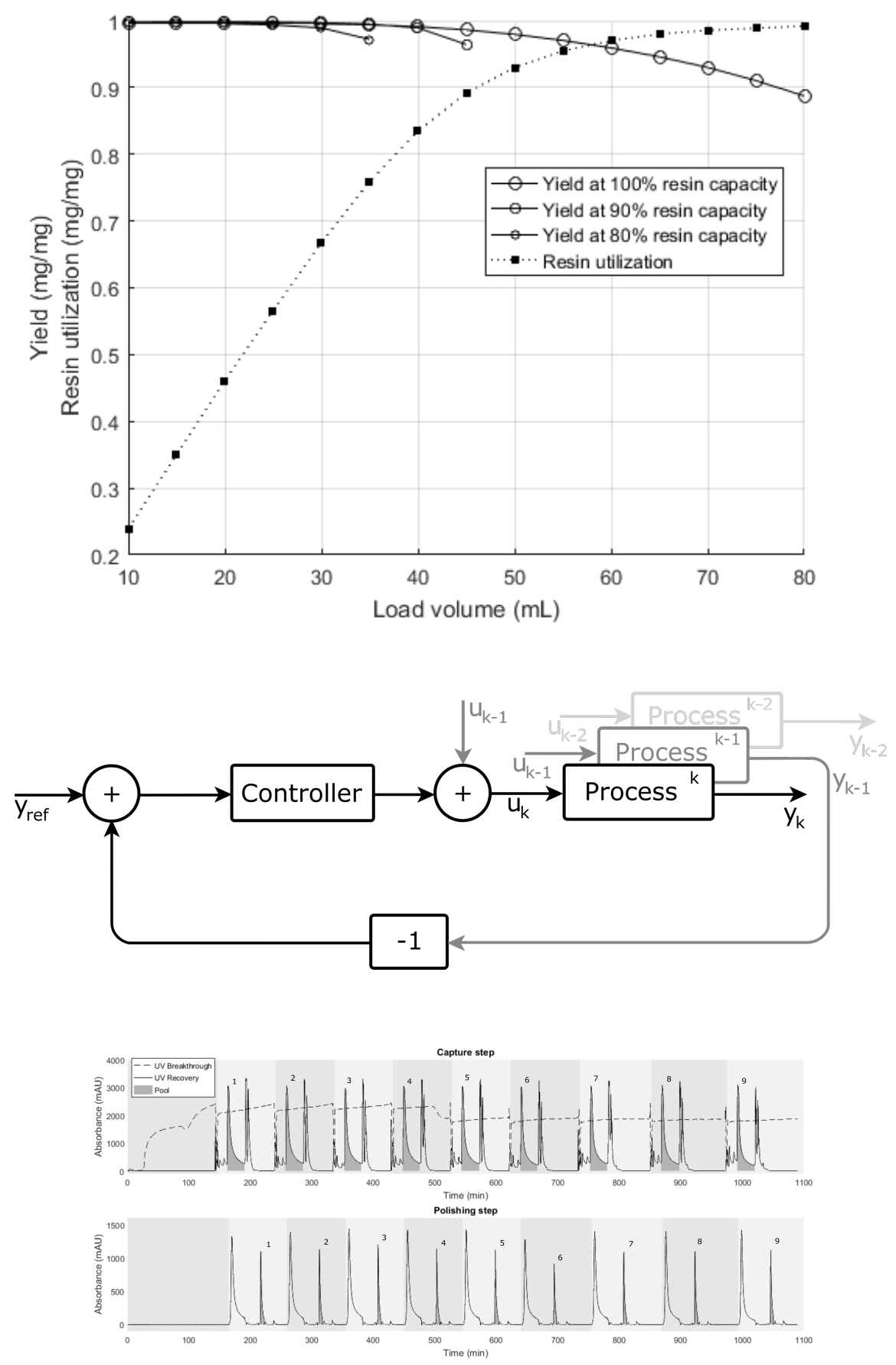

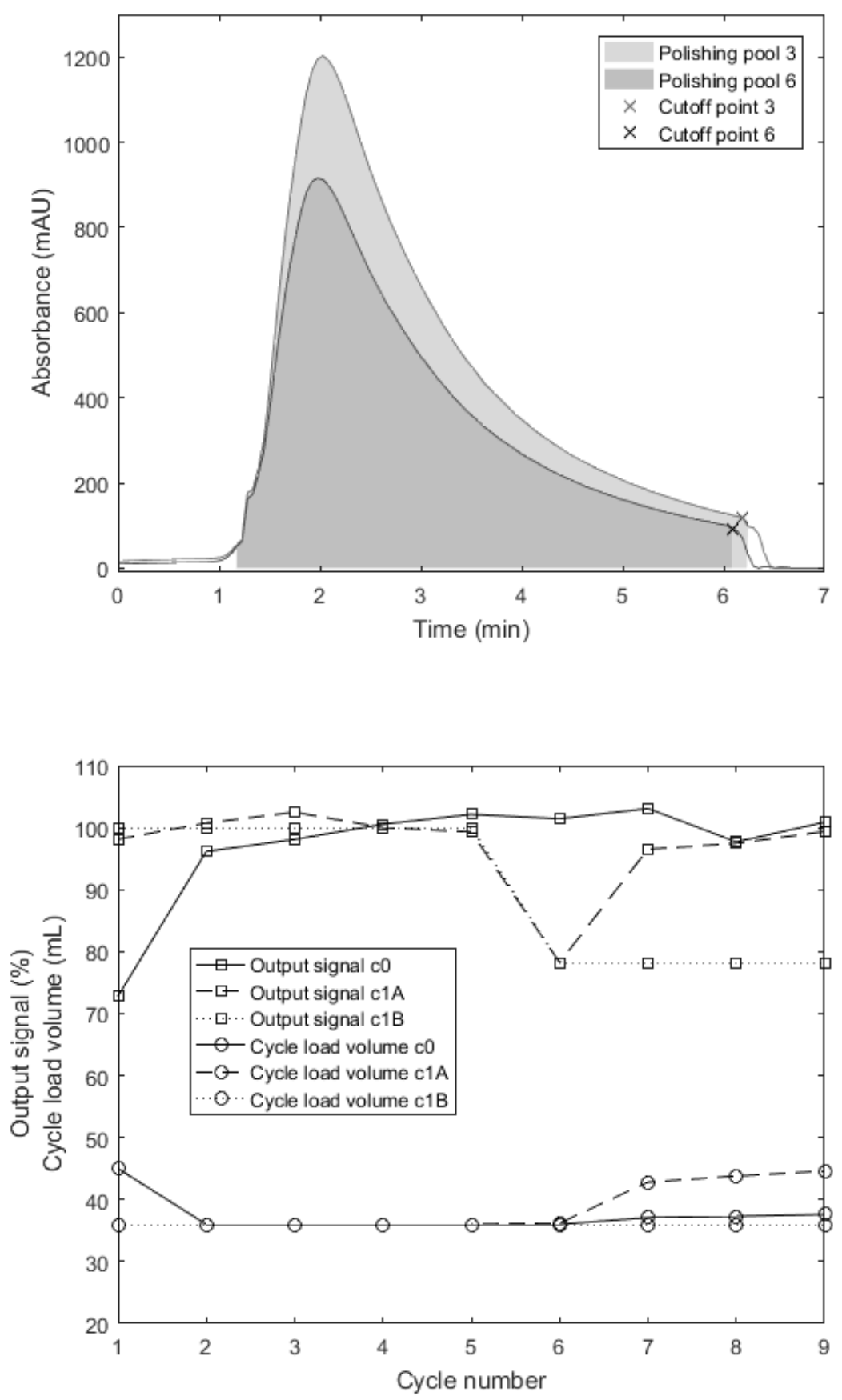